\section{Sensory impairments and cognitive disorders in older age}

Giancarlo Logroscino, Francesco Panza

Neurodegenerative Disease Unit,

Department of Basic Medicine,

Neuroscience, and Sense Organs,

University of Bari Aldo Moro; and

Department of Clinical Research in

Neurology, University of Bari

Aldo Moro, Pia Fondazione Cardinale

G. Panico, Tricase (LE), Italy

\section{Abstract}

Age-related and neuropathological changes in the olfactory, visual, auditory, and motor systems suggesting that sensory and motor changes may precede the cognitive symptoms of Alzheimer's disease (AD) by several years and may signify increase the risk of developing AD. In particular, peripheral age-related hearing impairment and social isolation have been identified as potentially modifiable dementia risk factors. The impact of age-related hearing and vision impairments on cognition appeared to be especially important among the oldest old suggesting a strong link of these connections with frailty, a critical intermediate status of the aging process at higher risk for negative health-related outcomes. The link among age-related hearing and vision impairments and cognition suggested the potential for correcting hearing and vision losses so that older subjects can function better cognitively with improved social involvement, quality of life, and lifetime cognitive health.

\section{Cognitive impairment, eye and ear}

In the last decade, the special senses, particularly hearing and vision, are key elements among the possible modifiable risk factors in building up dementia prevention strategies. Recently, the National Institute on Aging held an exploratory workshop focused on age-related and neuropathological changes in the olfactory, visual, auditory, and motor systems suggesting that sensory and motor changes may precede the cognitive symptoms of Alzheimer's disease (AD) by several years and may signify increase the risk of developing AD. ${ }^{1}$ The relationship between sensory impairment and cognitive impairment has been studied few times in population-based settings. The strongest association with cognition in older age has been found in several studies for hearing loss, and dementia research have focused on age-related hearing impairment or presbycusis as a possible risk factor for cognitive impairment and decline., ${ }^{2,3}$ In the last ten years, the cumulative evidence coming from a number of longitudinal population-based studies suggested a strong link between peripheral age-related hearing impairment and cognitive disorders in older age. ${ }^{2,3}$ Some cross-sectional studies have shown an association between vision impairment and late-life cognitive disorders, but in the older Australian cohort of the Blue Mountains Eye Study, although higher proportions of participants with visual, hearing or dual sensory impairment (hearing and vision) had a decline in global cognitive testing scores after 5 and 10 years compared to participants with no sensory impairment, no significantly greater risk of possible cognitive decline was found. ${ }^{4}$ The impact of age-related hearing and vision impairments on cognition appeared to be especially important among the oldest old suggesting a strong link of these connections with frailty, 2,3 a geriatric multidimensional syndrome characterized by a nonspecific state of vulnerability, reduced multisystem physiological reserve, and decreased resistance to stressors. Frailty is also an independent risk factor for cognitive impairment and dementia. ${ }^{2}$

It is possible that the association is not specific and not unique to one sensory system suggesting therefore that the presence of functioning sensory systems may be a marker of healthy brain aging as suggested recently by the investigators of the epidemiology of hearing loss study (EHLS), a longitudinal, population-based study of aging in the Beaver Dam, a community in Wisconsin..$^{5}$ In this study, hearing and vision impairments were associated with a relative risk around two with cognitive impairment while olfactory loss with a relative risk of four (5). In this study, however, $85 \%$ of participants with hearing impairment, $81 \%$ with visual impairment, and $76 \%$ with olfactory impairment did not develop cognitive impairment during follow-up. This selected risk for a segment of the population clearly indicated the presence of some other factors that may modulate the risk of cognitive impairment in presence of specific sensory deficits. ${ }^{5}$ A recent meta-analysis of studies on retinal thickness including 887 AD patients, 216 patients with mild cognitive impairment (MCI), and 864 healthy controls suggested a clear reduction of total macular thickness in patients with $\mathrm{AD}{ }^{6}$

Another layer of interest could be repre-
Correspondence: Giancarlo Logroscino, Neurodegenerative Disease Unit, Department of Basic Medical Sciences, Neurosciences, and Sense Organs, University of Bari Aldo Moro; and Department of Clinical Research in Neurology, University of Bari Aldo Moro, Pia Fondazione Cardinale G. Panico, Tricase, Lecce, Italy.

Tel.: +39.0833.773904;

Fax: +39.0833 .773909$

E-mail: giancarlo.logroscino@uniba.it

Key words: Presbycusis; age-related hearing loss; age-related vision impairment; Alzheimer's disease; dementia; frailty.

Conference presentation: paper presented at $31^{\circ}$ Congresso Nazionale SIGOT - Genova, June 8-9, 2017

Received for publication: 1 September 2017. Accepted for publication: 15 September 2017.

This work is licensed under a Creative Commons Attribution-NonCommercial 4.0 International License (CC BY-NC 4.0).

(C) Copyright G. Logroscino and F. Panza, 2017 Licensee PAGEPress, Italy

Geriatric Care 2017; 3:7039

doi:10.4081/gc.2017.7039

sented by new biomarkers for both early diagnosis and pathogenesis of disease. In the last few years, several ocular clinical symptoms and signs in $\mathrm{AD}$ have been reported: $\beta$-amyloid $(A \beta)$ accumulation in the retina and in the lens, fiber layer loss in the optic nerve, and especially, a wide range of retinal vascular pathological changes. Many of these changes have been proposed as potential biomarkers of $\mathrm{AD}$. One of the most interesting vascular markers is retinal blood flow. In a recent study, blood column diameter, blood speed, and blood flow have been measured in a major temporal retinal vein using retinal laser Doppler flowmetry in $\mathrm{AD}, \mathrm{MCI}$, and control subjects. In addition, peripapillary retinal nerve fiber layer (RNFL) thickness was measured using optical coherence tomography (OCT). Blood flow had intermediate values in MCI subjects between what was measured in controls and AD patients suggesting the importance of vascular changes in determining neurodegeneration. ${ }^{7}$ In patient with clinical $\mathrm{AD}$, the RNFL and macular thickness measured by spectral domain-OCT (SD-OCT) were significantly thinner compared to controls. ${ }^{8}$ The macula is thinner as well in all sectors except the fovea compared to controls. ${ }^{8}$ Macular measurements may be reliable indicators of visual impairment in $\mathrm{AD}$ and vision should be therefore checked in these patients. The most relevant issue is 
that visual dysfunction in $\mathrm{AD}$ patients can now be considered as direct outcome of retinal abnormalities due to the deposition of specific $A D$ pathology in the retina. Elevated $A \beta_{42 / 40}$ peptides, several types of $\mathrm{A} \beta$ plaques, and phospho-tau protein can be found in the retina with appropriate neuropathological techniques. ${ }^{9}$ In line with the above findings, in animal models have described retinal $A \beta$ deposits and tauopathy. ${ }^{9}$ As in specific areas of the brain the amyloid and tau deposits are associated with local inflammation, retinal ganglion cell degeneration may be an additional finding that could explain visual functional deficits in $\mathrm{AD} .^{10}$

\section{References}

1. Albers MW, Gilmore GC, Kaye J, et al. At the interface of sensory and motor dysfunctions and Alzheimer's disease. Alzheimer Dement 2015;11:70-98.

2. Panza F, Solfrizzi V, Logroscino G. Agerelated hearing impairment-a risk factor and frailty marker for dementia and AD. Nat Rev Neurol 2015;11:166-75.

3. Logroscino G, Panza F. The role of hearing impairment in cognitive decline: need for the special sense assessment in evaluating cognition in older age. Neuroepidemiol 2016;46:290-1.

4. Hong T, Mitchell P, Burlutsky G, et al. Visual impairment, hearing loss and cognitive function in an older population: longitudinal findings from the blue mountains eye study. PLoS One 2016; 11:e0147646.

5. Fischer ME, Cruickshanks KJ, Schubert $\mathrm{CR}$, et al. Age-related sensory impairments and risk of cognitive impairment. J Am Geriatr Soc 2016;64:1981-7.

6. den Haan J, Verbraak FD, Visser PJ, Bouwman FH. Retinal thickness in
Alzheimer's disease: A systematic review and meta-analysis. Alzheimers Dement (Amst) 2017;6:162-70.

7. Feke GT, Hyman BT, Stern RA, Pasquale LR. Retinal blood flow in mild cognitive impairment and Alzheimer's disease. Alzheimers Dement (Amst) 2015;1:144-51.

8. Polo V, Rodrigo MJ, Garcia-Martin E, et al. Visual dysfunction and its correlation with retinal changes in patients with Alzheimer's disease. Eye (Lond). 2017 [Epub ahead of print].

9. Frost S, Martins RN, Kanagasingam Y. Ocular biomarkers for early detection of Alzheimer's disease. J Alzheimers Dis 2010;22:1-16.

10. Williams PA, Thirgood RA, Oliphant H, et al. Retinal ganglion cell dendritic degeneration in a mouse model of Alzheimer's disease. Neurobiol Aging 2013;34:1799-806. 